\title{
Wavelet Transform-Based Approach to Defect Identification in Railway Carbon Contact Strips
}

\author{
Slawomir Judek ${ }^{1}$, Leszek Jarzebowicz ${ }^{1}$ \\ ${ }^{1}$ Faculty of Electrical and Control Engineering, Gdansk University of Technology, \\ G. Narutowicza 11/12, 80-233 Gdansk, Poland \\ slawomir.judek@.pg.gda.pl
}

\begin{abstract}
Pantographs of electric rail vehicles are fitted with carbon contact strips, which slide along the contact wire of catenary to provide constant electrical contact. Contact strips are exposed to wear and damages. Using damaged contact strips significantly increases the risk of catenary rupture. Therefore, their technical condition has to be inspected frequently. In previous work a 3D laser scanning system was proposed for recording contact strips surface shape when vehicles pass an inspection point on a railway line. The system was complimented with an effective automatic wear estimation algorithm. However, this algorithm is insensitive to some shallow defects which are considered hazardous. This paper presents defects identification approach based on differentiation of contact strips profiles. It is assumed that the hazard of catenary rupture corresponds to the peak value in the differentiated profile. A wavelet transform was used to allow for robust differentiation of profiles featured by low signal-tonoise ratio. A method for choosing well-balanced dilatation parameter was proposed, in order to obtain good accuracy of differentiation of both smooth and narrow-edged contact strip defects. Results of simulations implemented in Matlab confirm that the proposed approach can be applied to an algorithm aiming for automatic defects identification.
\end{abstract}

Index Terms-Condition-based maintenance, current collector, fault diagnosis, pantograph, railway transportation.

\section{INTRODUCTION}

Reliability and safety are crucial in railway transportation. Therefore, increased interest can be noticed in various maintenance approaches [1]-[3]. Current collection system, containing catenary and pantographs, is one of maintenance concerns. Typical pantographs time-based preventive maintenance takes place during periodic services performed in rolling stock depots. Such an approach is time consuming, imprecise, human factor sensitive and cost ineffective [4]. Therefore, condition-based maintenance has recently gained on importance [1]. It is based on inspection points located on railway lines to perform diagnostics on running vehicles.

Pantographs are fitted with a pair of Carbon Contact Strips (CCS) which slide along the contact wire of the catenary to provide electrical contact (see Fig. 1). CCS are exposed to wear and damages, as shown in Fig. 1(b) and Fig. 1(c), respectively. Overly worn or damaged CCS have to be promptly detected and replaced, since they cause disturbances of current collection and increase the rate of contact wire wear. Moreover, contact wire may hinder on a defect while staggering across the strip and result in catenary rupture.

This paper is related to previous works [4], [5], which concern CCS diagnostics. In [4] authors proposed a new Laser Scanning Inspection System (LSIS) for CCS diagnostics. Afterwards, they complimented the system with an automatic wear estimation algorithm based on the 3D data processing [5]. Experimental tests confirmed good performance of this algorithm. However, the method is insensitive to shallow defects, like the one shown in Fig. 1(c). Such defects need to be identified in terms of reducing catenary rupture hazard.

In the paper we propose an approach for CCS defects identification designated for LSIS. We assume that the defects can be detected by differentiation of $z=f(x)$ CCS profiles (see Fig. 1 for axes labelling). We use Wavelet Transform (WT) to compute the $\mathrm{d} z / \mathrm{d} x$ from LSIS measurements. A pattern of CCS defects was introduced in order to select WT dilatation parameter and validate the proposed approach by the means of computer simulation.

\section{RELATED WORKS}

Pantographs may be equipped with the means of detecting CCS faults. Methods based on pneumatic dropping devices and electronic sensors are proposed in [6] and [7].

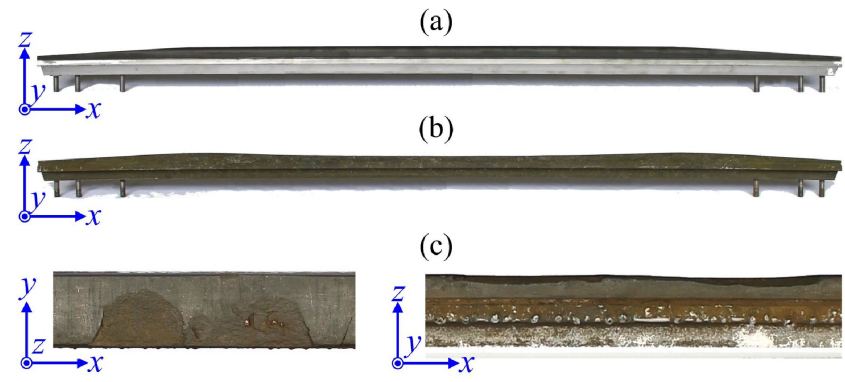

Fig. 1. CCS of a various condition: (a) new, (b) worn, (c) chipped.

Another approach of CCS diagnostics relies on inspection points located on railway lines. Vision systems seem to be the convenient tool to monitor the shape of a carbon layer surface. A solution which incorporates several cameras and linear laser illuminators is reported in [8]. The proposed image analysis algorithm allows for defining selected CCS dimensions and assesses the losses of carbon. In turn, in [9] 
the authors describe a system which consists of only two cameras. The first one is applied to inspect CCS and the second serves to assess the collector head horns. By the image analysis CCS wear is estimated and notches are detected. The reported experiments show that, on average, in $20 \%$ of cases it is not possible to make a correct diagnosis of the contact strips.

Finally, the most common approach to CCS diagnostics is manual inspection. The criteria of evaluation of CCS technical condition are not included in the standards, e.g. EN 50206, EN 50405 or Technical Specifications for Interoperability of railway systems. Some recommendations can be found in operating manuals provided by CCS manufacturers [10].

\section{PROBLEM Formulation AND MAIN CONTRIBUTION}

Experience of rolling stock service indicates that there are numerous cases of CCS defects, mainly carbon layer chipping. LSIS is expected to detect such faults. Since not all these defects are deep enough to be identified as an excessive wear, an additional algorithm has to be applied exclusively for defects detection and identification.

As one can see from the review of related works the only algorithm aiming for defects detection is reported in [8]. However, the general description does not allow for reconstruction of the proposed method. Moreover, there is a lack of evaluation of the detected defects in order to estimate the hazard of catenary breakage. In turn, the recommendations included in CCS manuals for periodical inspection cannot be straightway transferred to the defects identification algorithm, because they are rather descriptive than numerical. Therefore, a specific algorithm is needed to enable LSIS to identify the defects.

The aim of the paper is to introduce, set up and validate an algorithm for defects detection and identification designated for LSIS. The main objective of this algorithm is to detect defects which may hinder the contact wire when staggering across the strip. The defects should be evaluated in a scale indicating the hazard probability.

We propose an algorithm which is based on differentiation of CCS height profiles $z=f(x)$. The edges of CCS defects result in the peaks in $\mathrm{d} z / \mathrm{d} x$ function. The peaks magnitudes depend on the steepness of the edges. Assuming the steepness as hazard-indicating parameter, defect identification can be performed by finding the maximums of the module $|\mathrm{d} z / \mathrm{d} x|$ and comparing it with some predefined scale. This scale can be established afterwards, based on exploitation practice.

Although the method is simple, differentiation of the measured $z=f(x)$ profile can be troublesome due to the low signal-to-noise ratio (SNR). Therefore, the differentiation method has to apply filtering, and to be adjusted in such a way to enable the algorithm to accurately estimate both gentle and steep slopes.

The main contribution of the paper is applying a wavelet transform based algorithm for CCS defects detection and identification. The algorithm was implemented in Matlab. The method was evaluated and validated throughout computer simulation based on a proposed standard of CCS damage.

\section{PRINCIPLE OF DIFFERENTIATION METHOD FOR MONITORING CCS PROFILES}

Firstly numeric results of CCS scanning as heights expressed in millimeters are collected. They form a matrix whose rows and columns correspond to $x$ and $y$ coordinates, respectively. Each matrix row contains 1536 heights, measured in evenly spaced points, spread along the $x$ axis. Such a set of points form discrete $z=f(x)$ function.

An exemplary result of differentiation by computing difference quotient $\Delta z / \Delta x$ for subsequent pairs of samples was shown in Fig. 2. Such an approach cannot be directly applied to the LSIS measurements to detect and identify the defects because of low SNR. To improve the SNR, digital signal smoothing is required. Typical differentiation methods include averaging, least-squares polynomial smoothing of Savitzky-Golay, spline, and Fourier Transform are commonly used [11], [12]. However, the use of these methods encounters difficulties in adjusting parameters. Furthermore it involves complex calculations and is dedicated only to applications with the high SNR. Besides the conventional methods, a wavelet transform is also proposed for similar applications [12], [13].

The wavelet transform $W f(a, b)$ of a function $z=f(x)$ at the dilatation $a$ and translation $b$ parameters is computed by correlating $f(x)$ with a wavelet atom $\psi_{a, b}(x)$

$$
W f(a, b)=\int_{-\infty}^{+\infty} f(x) \frac{1}{\sqrt{a}} \psi *\left(\frac{x-b}{a}\right) \mathrm{d} x
$$

One of the most important properties of the wavelet transform is the vanishing moment. Function $\psi(x)$ has $n$ vanishing moments which satisfy the following condition

$$
\int_{-\infty}^{+\infty} x^{k} \psi(x) \mathrm{d} x=0
$$

where $0 \leq k<n$. A wavelet $\psi(x)$ with fast decay has $n$ vanishing moments if and only if there exists a function $\theta(x)$ such that

$$
\psi(x)=(-1)^{n} \frac{\mathrm{d}^{n} \theta(x)}{\mathrm{d} x^{n}},
$$

where $\theta(x)$ is a function with fast decay and whose integral is a nonzero constant, e.g. satisfies the condition of a smoothing function and a consequence

$$
W f(a, b)=a^{n} \frac{\mathrm{d}^{n}}{\mathrm{~d} b^{n}}\left(f * \overline{\boldsymbol{\theta}}_{a}\right)(b),
$$

with

$$
\bar{\theta}_{a}(x)=\frac{1}{\sqrt{a}} \theta\left(-\frac{x}{a}\right) .
$$

Hence, a wavelet transform $W f(a, b)$ can be denoted as the 
$n^{\text {th }}$-order derivative of a smooth function $f(x)$ over a domain proportional to parameter $a$ [12], [13]. Exemplary results of differentiation using WT are shown in Fig. 2.

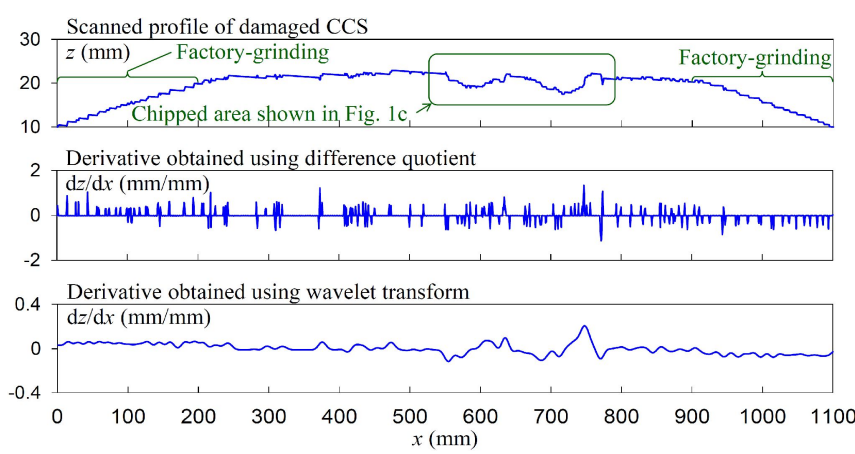

Fig. 2. Measured profile of damaged CCS, presented previously in Fig. 1(c), and results of its differentiation.

\section{IMPLEMENTATION, EVALUATION AND VERIFICATION OF WT-BASED DIFFERENTIATION ALGORITHM}

Accordingly to the algorithm objectives, the function $\mathrm{d} z / \mathrm{d} x$ computed for a damaged CCS needs to clearly reveal its maximums and minimums corresponding to the edges of defects. Moreover, their magnitudes have to be fairly precise to allow for the hazard evaluation of catenary rupture. The error $e_{d}$ of the algorithm was defined as a difference between the estimated and real derivatives, computed for the maximum and minimum of the function $\mathrm{d} z / \mathrm{d} x$.

The shape of usual defects is highly irregular. Calculating the reference function $\mathrm{d} z / \mathrm{d} x$ in order to obtain error $e_{d}$ would be troublesome. Therefore, a standardized model of CCS damages is proposed to validate the differentiation method throughout computer simulations.

The steepness of defect edges varies in a wide range. The error $e_{d}$ depends on dilatation parameter $a$. High value of the parameter suppresses noise, but on the other hand it flattens narrow damages. In turn, a low dilatation decreases the filtering and increases the risk that the detected maximum or minimum of $\mathrm{d} z / \mathrm{d} x$ would identify just a noise peak. Therefore, it is important to optimize dilatation parameter in order to balance the error for the whole range of assumed edge steepness. Moreover, error $e_{d}$ is to be computed for the selected dilatation parameter to ultimately evaluate the proposed approach.

\section{A. A Damaged CCS Test Profile}

The proposed test profiles are based on a desire trapezoidal shape corresponding to a factory new CCS [5]. The desire trapezoidal profile is combined with defect pattern, located in the middle. Due to the lack of damage standardization the test profile has to contain typical damages, described using simple mathematical formulas and parameters. We propose a co-sinusoidal-shaped pattern, shown in Fig. 3 and given by

$$
z_{\text {def }}(x)=H-A+A \cos \left(\frac{2 \pi}{T}\left(x-\frac{c}{2}\right)\right),
$$

where $H$ is the height of the middle-part of a factory new contact strip, $A$ is half of the defect depth, $T$ is the defect width, and $c$ is the total CCS width.
Differentiation of (6) in respect to $x$ results in a simple continuous function

$$
\frac{\mathrm{d} z_{d e f}}{\mathrm{~d} x}=-\frac{2 \pi A}{T} \sin \left(\frac{2 \pi}{T}\left(x-\frac{c}{2}\right)\right) .
$$

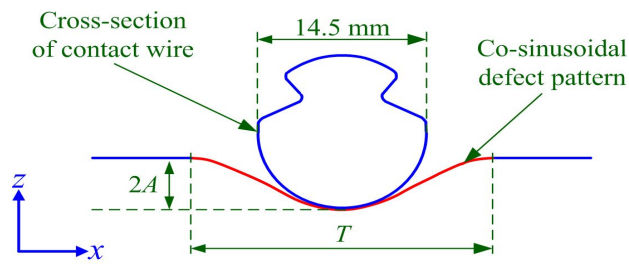

Fig. 3. A front cut of the proposed pattern of CCS test defect.

The test profile consisting of a standardized damage is contaminated with noise in order to evaluate model properties. Gaussian noise was proposed with a level being twice of that registered by LSIS. Graphs of the CCS profile model, with and without the noise, are presented in Fig. 4(a).

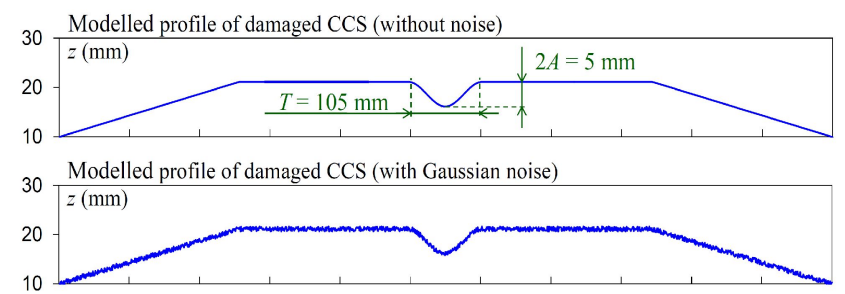

a)

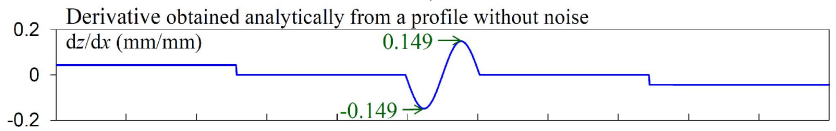

0.4 Derivative obtained using wavelet transform for dilatation $a=2$

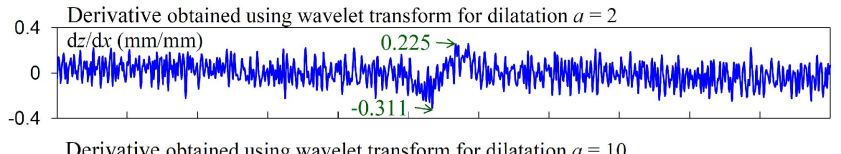

0.4 Derivative obtained using wavelet transform for dilatation $a=10$

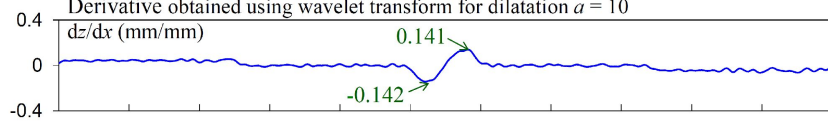

0.4 Derivative obtained using wavelet transform for dilatation $a=48$

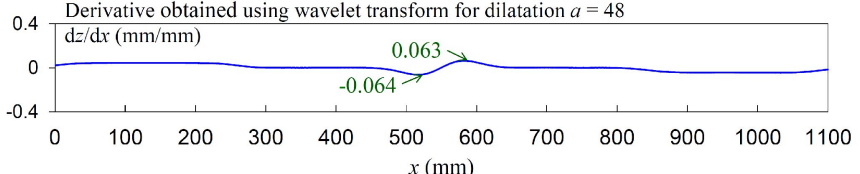

b)

Fig. 4. An example of modelling a); and analysis (b) of a damaged CCS profile.

\section{B. Evaluation of the Method}

To find how the dilatation parameter $a$ affects an error $e_{d}$ for different defect dimensions, CCS test profile was subjected to the WT-based differentiation algorithm implemented in Matlab.

The relation between error $e_{d}$ and dilatation parameter $a$ was investigated for various depths $A$ and widths $W$ of the defect. Two values of the damage depth were considered. A case of $2 A=5 \mathrm{~mm}$ corresponds to the shallowest damage which is expected to be identified. The second case of $2 A=10 \mathrm{~mm}$ was included to verify how much the depth influences the error. Width $T$ of the damage was varied by six levels. It was assumed that the most narrow damage which may hinder the contact wire has a size of $T=26 \mathrm{~mm}$. 
The widest applied damage pattern has a size of $T=587 \mathrm{~mm}$ which corresponds to the width of a flat part of factory new CCS. The latter case is a model of worn CCS. Dilatation parameter $a$ was varied in a range from 2 to 48 what results with peak values of $\mathrm{d} z / \mathrm{d} x$ from $0.0267 \mathrm{~mm} / \mathrm{mm}$ to $1.19 \mathrm{~mm} / \mathrm{mm}$.

The graphs of the function $\mathrm{d} z / \mathrm{d} x$ computed for selected dimensions of the damage are shown in Fig. 4(b). The maximum and minimum values, marked in the figure, differ for each of considered dilatation parameter settings.

The characteristics for $2 A=5 \mathrm{~mm}$ and $2 A=10 \mathrm{~mm}$ are shown in Fig. 5 and Fig. 6, respectively. The relation between error $e_{d}$ and dilatation parameter $a$ is monotonous but not linear. The absolute error of estimating minimum and maximum $\mathrm{d} z / \mathrm{d} x$ is similar for particular widths and depths of the damage. The influence of the damage depth on the error is visible only for low values of $a$.
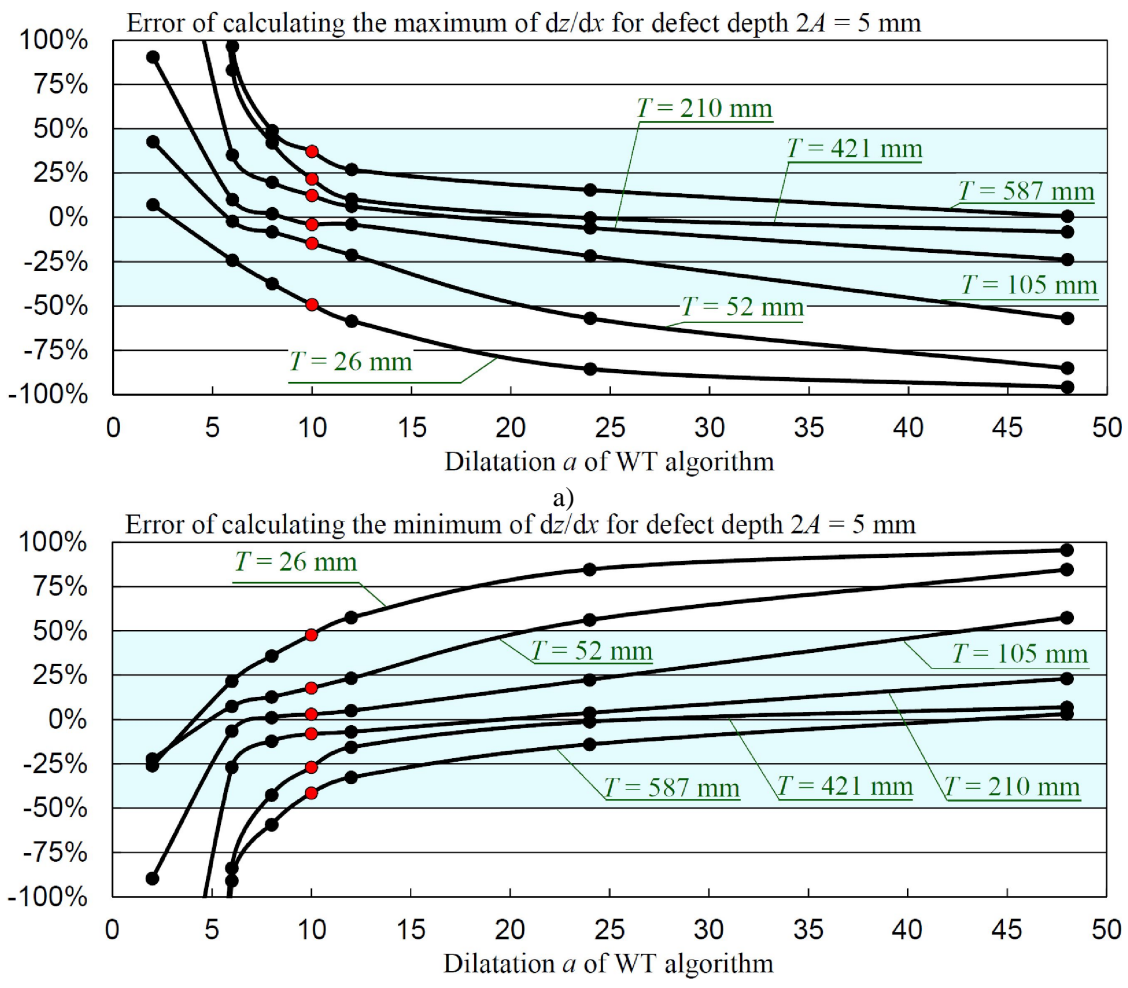

b)

Fig. 5. Error characteristics of estimating maxima a) and minima b) for a CCS profile model with defect depth of $2 A=5 \mathrm{~mm}$ for different $T$.
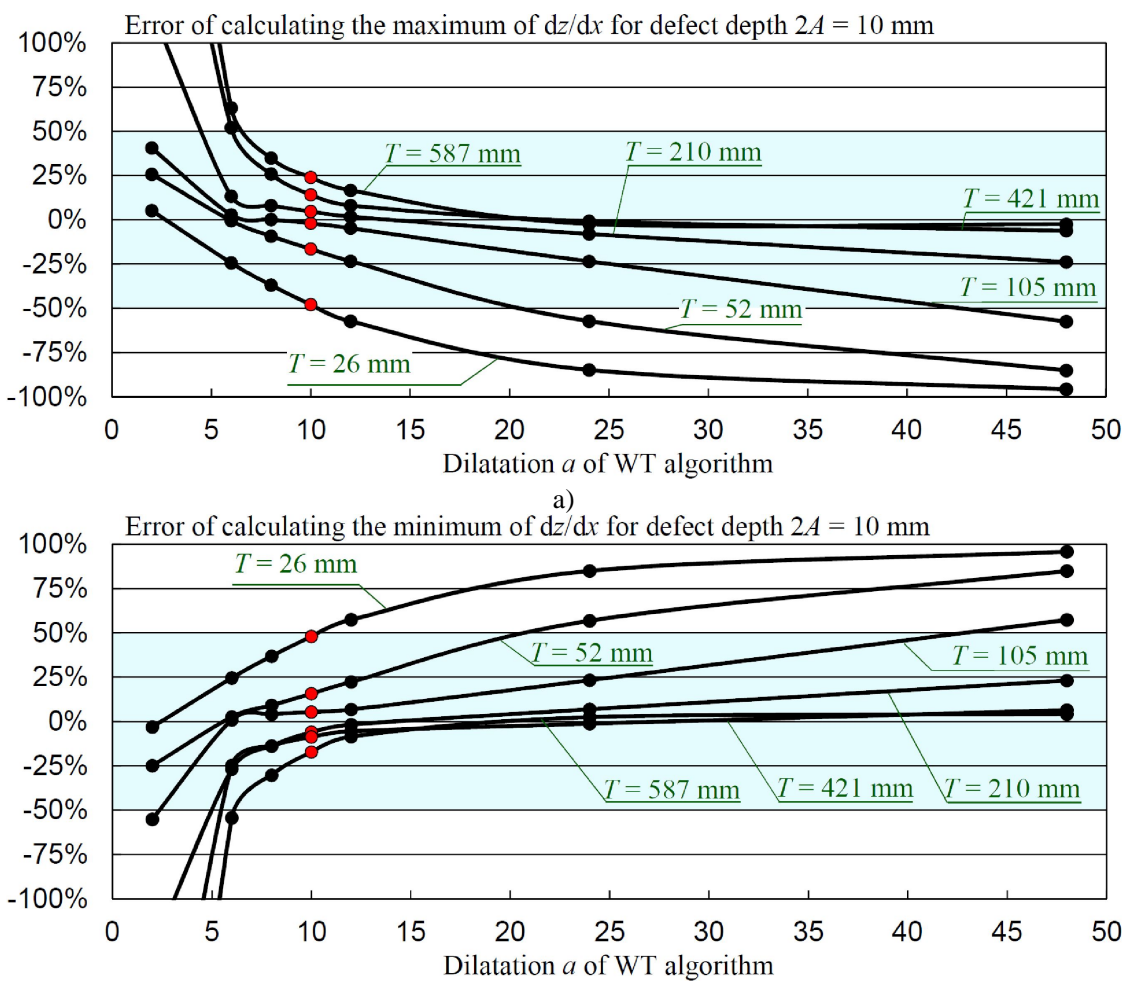

b)

Fig. 6. Error characteristics of estimating maxima a) and minima b) for a CCS profile model with defect depth of $2 A=10 \mathrm{~mm}$ for different $T$. 
Considering all the variables, the minimal error value for the assumed damage widths and depths was obtained for $a=10$. In such a case error $e_{d}$ is less than $50 \%$.

\section{CONCLUSION}

A pioneer approach to automatic CCS defects identification was proposed. The approach is based on differentiating CCS profiles and seeking the maximal and minimal $\mathrm{d} z / \mathrm{d} x$.

WT-based algorithm allows for differentiating noisy CCS profiles. The filtering proprieties of the algorithm depend on a single parameter which simplifies the process of tuning.

Detection and rough evaluation of defects does not require very precise $\mathrm{d} z / \mathrm{d} x$ estimation. However, a sensible level of uncertainty has to be assured for a wide range of estimated slope rates. The obtained error of value less than $50 \%$ is sufficient to meet the requirements of defects identification.

The proposed approach will be applied to LSIS. A set of damaged CCS is being collected thanks to the courtesy of rolling stock operators. This will allow for validation of the proposed approach on the basis of real CCS profiles.

\section{ACKNOWLEDGMENT}

The authors would like to thank Dr Wlodek J. Kulesza from Blekinge Institute of Technology in Sweden for the constructive comments that helped to improve the presentation of the paper.

\section{REFERENCES}

[1] A. K. S. Jardine, D. Lin, D. Banjevic, "A review on machinery diagnostics and prognostics implementing condition-based maintenance", Mech. Syst. Signal Process, vol. 20, no. 7, pp. 14831510, 2006. [Online]. Available: http://dx.doi.org/10.1016/ j.ymssp.2005.09.012

[2] A. Schobel, T. Maly, "Operational fault states in railways", Eur. Transp. Res. Rev., vol. 4, no. 2, pp. 107-113, 2012. [Online] Available: http://dx.doi.org/10.1007/s12544-011-0068-Z

[3] M. Bartlomiejczyk, S. Hamacek, D. Kolosov, Y. Zharkov, "Automated diagnostics of current pick-up disturbances in electric traction networks", in Proc. 2014 14th Int. Conf. on Environment and Electrical Engineering (EEEIC), 2014, pp. 378-381.

[4] L. Jarzebowicz, S. Judek, "3D machine vision system for inspection of contact strips in railway vehicle current collectors", in Proc. 2014 Int. Conf. on Applied Electronics (AE), 2014, pp. 139-144. [Online]. Available: http://dx.doi.org/10.1109/AE.2014.7011686

[5] S. Judek, L. Jarzebowicz, "Algorithm for automatic wear estimation of railway contact strips based on 3D scanning results", in Proc. 2014 Int. Conf. and Exposition on Electrical and Power Engineering (EPE), 2014, pp. 724-729. [Online]. Available: http://dx.doi.org/ 10.1109/icepe.2014.6970004

[6] A. Steimel, Elektrische Triebfahrzeuge und ihre Energieversorgung: Grundlagen und Praxis. Germany: Oldenbourg Industrieverlag, 2014

[7] Intelligent Carbon for Pantographs, Schunk Bahn- und Industrietechnik GmbH, 2012. [Online]. Available: http://www.schunk-sbi.com

[8] E. C. W. Kin, "Pioneer design in automatic pantograph wear monitoring", Engineering Integrity, vol. 19, pp. 12-17, 2006.

[9] L. G. C. Hamey, T. Watkins, S. W. T. Yen, "Pancam: in-service inspection of locomotive pantographs", in Proc. 9th Biennial Conf. the Australian Pattern Recognition Society on Digital Image Computing Techniques and Applications, 2007. [Online]. Available: http://dx.doi.org/10.1109/dicta.2007.4426837

[10] Carbon contact strips service instructions, Morgan Carbon, 2011 , unpublished.

[11] P. H. C. Eilers, "A perfect smoother", Anal. Chem., vol. 75, no. 14, pp. 3631-3636, 2003. [Online]. Available: http://dx.doi.org/10.1021 /ac034173t

[12] X. Shao, C. Ma, "A general approach to derivative calculation using wavelet transform", Chemom. Intell. Lab. Syst., vol. 69, no. 1-2, pp. 157-165, 2003. [Online]. Available: http://dx.doi.org/10.1016/ j.chemolab.2003.08.001

[13] S. Mallat, A Wavelet Tour of Signal Processing. The Sparse Way. Amsterdam, Boston: Academic Press, 2008. 